\title{
Optimized Explicit Runge-Kutta Schemes for Entropy Stable Discontinuous Collocated Methods Applied to the Euler and Navier-Stokes equations
}

\author{
R. Al Jahdali *, R. Boukharfane ${ }^{\dagger}$, L. Dalcin ${ }^{\dagger}$, and M. Parsani ${ }^{\S}$ \\ King Abdullah University of Science and Technology (KAUST), Computer Electrical and Mathematical Science and \\ Engineering Division (CEMSE), Extreme Computing Research Center (ECRC), 23955-6900, Thuwal, Saudi Arabia
}

In this work, we design a new set of optimized explicit Runge-Kutta schemes for the integration of systems of ordinary differential equations arising from the spatial discretization of wave propagation problems with entropy stable collocated discontinuous Galerkin methods. The optimization of the new time integration schemes is based on the spectrum of the discrete spatial operator for the advection equation. To demonstrate the efficiency and accuracy of the new schemes compared to some widely used classic explicit Runge-Kutta methods, we report the wall-clock time versus the error for the simulation of the two-dimensional advection equation and the propagation of an isentropic vortex with the compressible Euler equations. The efficiency and robustness of the proposed optimized schemes for more complex flow problems are presented for the three-dimensional Taylor-Green vortex at a Reynolds number of $\operatorname{Re}=$ $1.6 \times 10^{3}$ and Mach number $\mathrm{Ma}=0.1$, and the flow past two identical spheres in tandem at a Reynolds number of $\mathrm{Re}=3.9 \times 10^{3}$ and Mach number $\mathrm{Ma}=0.1$.

\section{Introduction}

Spatially high-order accurate discretizations demonstrate much smaller numerical errors than low-order methods, in terms of dispersion as well as dissipation [1-4]. Consequently, they tend to be more accurate per degree of freedom (DOF) $[5,6]$. In addition, from a high performance computing perspective, compact high-order accurate schemes are very good candidates for the current and next-generation hardware, due to their smaller communication-to-computation ratios; see, for example, the works of Hutchinson et al. [6], Vincent et al. [7], Hadri et al. [8], Fischer et al. [5], and Merzari et al. [9]. However, high-order spatial discretizations suffer from numerical instabilities when the flow contains discontinuities (e.g., shocks) or under-resolved physical features (e.g., turbulence structures). As a result, during the last two decades, starting from the work of Tadmor [10], a lot of effort has been devoted to the design of high-order entropy stable methods for compressible flows. The discrete entropy stability framework of [10], closely mimics some important nonlinear stability properties of the continuous system of nonlinear partial differential equations. The extension of Tadmor [10] and LeFloch ideas [11] to general high-order accurate discretizations has led to the development of a wide range of spatial discretizations [11-21] that can be proved to be nonlinearly stable (entropy stable) as long as positivity of the thermodynamic variables is preserved. Noticeably, high-order entropy stable algorithms offer a high level of robustness [22, 23] and, when carefully implemented, scale well on large supercomputers [24].

When numerically solving partial differential equations, stability constraints often limit the time step size of classical temporal methods when used in combination with spatially high-order accurate algorithms. Therefore, substantial effort

\footnotetext{
*Postdoctoral research fellow, rasha.aljahdali@kaust.edu.sa, AIAA member

$\dagger$ Postdoctoral research fellow, radouan.boukharfane@kaust.edu.sa, AIAA member

†Research Scientist, dalcinl@gmail.com, AIAA member

$\S$ Assistant Professor, matteo.parsani@kaust.edu.sa, AIAA member
} 
has been dedicated to the design of time integration schemes that admit larger stable time step sizes, while retaining a small local truncation error [25-30], and a small memory footprint [26, 27]. Runge-Kutta methods (RK) are one of the most widely used family of methods applied to initial value problems. The stability polynomial of a RK methods controls its linear stability and accuracy [31,32]. To enlarge the absolute stability regions of a time integration scheme, numerous techniques that encapsulate better the spectrum of a spatial discretizations have been developed [25, 27, 28]. The focus of this work is on the design of optimized explicit Runge-Kutta (ERK) schemes for the integration of systems of ordinary differential equations that arise from the spatial discretization with entropy stable high-order collocated discontinuous Galerkin methods on tensor product elements. Summation-by-part (SBP) operators constructed on the Legendre-Gauss-Lobatto (LGL) points $[13,14,33-36]$ are at the core of the spatial discretizations implemented in the scalable SSDC framework [37].

The paper is organized as follows. In Section II, an overview of the spatial discretization and the description of the partial differential equations used to generate the spectra for the ERK optimization procedure are given. In Section III, the description of the optimization process used to construct the new ERK is provided. Section IV is devoted to the performance study of the proposed methods, whereas Section $\mathrm{V}$ presents the performance analysis of the schemes for two viscous flow problems of increasing complexity. Finally, the conclusions are drawn in Section VI.

\section{Preliminaries}

In this work, the spatial discretization is performed using entropy stable discontinuous collocated Galerkin methods based on summation-by-parts and simultaneous approximation term operators [13, 15, 18, 38-40]. The one-dimensional SBP operators are applied to discretize the derivatives in each element. These operators can be defined as the following $[41,42]$.

Definition 1 A matrix operator, $\mathrm{D}_{\xi} \in \mathbb{R}^{N \times N}$, is an SBP operator of degree $p$ approximating the derivative $\frac{\partial}{\partial \xi}$ on the domain $\xi \in[\alpha, \beta]$ with nodal distribution $\xi$ having $N_{l}$ nodes, if

1) $\mathrm{D}_{\xi} \boldsymbol{\xi}^{j}=j \boldsymbol{\xi}^{j-1}, j=0,1, \ldots, p$;

2) $\mathrm{D}_{\xi} \equiv\left(\mathrm{P}_{\xi}\right)^{-1} \mathrm{Q}_{\xi}$, where the norm matrix, $\mathrm{P}_{\xi}$, is symmetric positive definite;

3) $\mathrm{Q}_{\xi} \equiv\left(\mathrm{S}_{\xi}+\frac{1}{2} \mathrm{E}_{\xi}\right), \mathrm{S}_{\xi}=-\left(\mathrm{S}_{\xi}\right)^{\mathrm{T}}, \mathrm{E}_{\xi}=\left(\mathrm{E}_{\xi}\right)^{\mathrm{T}}$, $\mathrm{E}_{\xi}=\operatorname{diag}(-1,0, \ldots, 0,1)=\boldsymbol{e}_{N} \boldsymbol{e}_{N}^{\mathrm{T}}-\boldsymbol{e}_{1} \boldsymbol{e}_{1}^{\mathrm{T}}, \boldsymbol{e}_{1} \equiv[1,0, \ldots, 0]^{\mathrm{T}}$, and $\boldsymbol{e}_{N} \equiv[0,0, \ldots, 1]^{\mathrm{T}}$.

A degree $p$ SBP operator is hence one that differentiates exactly monomials up to degree $p$.

In this work, the SBP operators are extended to multiple dimensions by using tensor products $(\otimes)$.

\section{A. Spatial discretization of hyperbolic conservation laws}

Consider a general system of hyperbolic conservation laws written using the three-dimensional Cartesian coordinates system

$$
\begin{cases}\frac{\partial \boldsymbol{q}}{\partial t}+\sum_{m=1}^{3} \frac{\partial \boldsymbol{F}_{x_{m}}}{\partial x_{m}}=0, & \forall\left(x_{1}, x_{2}, x_{3}\right) \in \Omega, \quad t \geq 0, \\ \boldsymbol{q}\left(x_{1}, x_{2}, x_{3}, t\right)=g^{(B)}\left(x_{1}, x_{2}, x_{3}, t\right), & \forall\left(x_{1}, x_{2}, x_{3}\right) \in \partial \Omega, \quad t \geq 0, \\ \boldsymbol{q}\left(x_{1}, x_{2}, x_{3}, 0\right)=g^{(0)}\left(x_{1}, x_{2}, x_{3}, 0\right), & \forall\left(x_{1}, x_{2}, x_{3}\right) \in \Omega,\end{cases}
$$

where the vectors $q$ and $\boldsymbol{F}_{x_{m}}$ are the conserved variables and the inviscid fluxes, respectively, and $g^{(0)}$ and $g^{(B)}$ represent the initial and boundary conditions. The approximation of the conservation law system (1) starts with the division of the 
domain $\Omega$ into $K$ non-overlapping elements. Using tensor-product elements, we can discretize the physical domain $\Omega \subset \mathbb{R}^{3}$ with boundary $\partial \Omega$. The generic (linear or entropy) stable discretization of (1) on the $\kappa^{\text {th }}$ element, written as:

$$
\frac{\mathrm{d} \boldsymbol{q}_{\kappa}}{\mathrm{d} t}+\sum_{m=1}^{3} 2 \mathrm{D}_{x_{m}}^{I, \kappa} \circ \mathrm{F}_{x_{m}}\left(\boldsymbol{q}_{\kappa}, \boldsymbol{q}_{\kappa}\right) \mathbf{1}_{\kappa}=\mathbf{S A T}^{\mathrm{I}}+\mathbf{S A T}^{\mathrm{BC}}+\mathbf{d i s s}^{I}
$$

where the vector $\boldsymbol{q}_{\kappa}$ is the discrete solution at the collocated nodes, and the vectors diss ${ }^{I}$ is added interface dissipation (the construction of this is detailed in $[13,24]$ ). The $\mathbf{S A T} \mathbf{T}^{\mathrm{I}}$ and $\mathbf{S A} \mathbf{T}^{\mathrm{BC}}$ are utilized to weakly couple neighboring elements and impose boundary conditions, respectively. The specific construction of the interface coupling term, SAT ${ }^{\mathrm{I}}$, depends on whether the interface is conforming or nonconforming. For more details on conforming interfaces, please refer to [33], and for nonconforming interfaces refer to [39]. Equation (2) shows that the inviscid flux derivatives are approximated in a particular way, i.e.,

$$
\frac{\partial \boldsymbol{F}_{x_{m}}}{\partial x_{m}} \approx 2 \mathrm{D}_{x_{m}}^{I, \kappa} \circ \mathrm{F}_{x_{m}}\left(\boldsymbol{q}_{\kappa}, \boldsymbol{q}_{\kappa}\right) \mathbf{1}_{\kappa}
$$

where $\mathrm{D}_{x_{m}}^{I, \kappa}$ is a differentiation matrix for the $x_{m}$ direction, $\circ$ is the Hadamard product (entry-wise multiplication), $\mathrm{F}_{x_{m}}\left(\boldsymbol{q}_{\kappa}, \boldsymbol{q}_{\kappa}\right)$ is a two-point flux function matrix (the construction of this matrix is detailed in [24]), and $\mathbf{1}_{\kappa}$ is a vector of ones. Approximation (3) enables us to mimic the continuous nonlinear (entropy) stability analysis at the semi-discrete level [36]. The system of conservation laws (1) is solved in computational space in the SSDC solver. The differentiation matrix $\mathrm{D}_{x_{m}}^{I, \kappa}$ is therefore constructed as the following:

$$
\mathrm{D}_{x_{m}}^{I, \kappa} \equiv \frac{1}{2} J_{\kappa}^{-1} \sum_{l=1}^{3}\left(\mathrm{D}_{\xi_{l}}\left[\mathcal{J} \frac{\partial \xi_{l}}{\partial x_{m}}\right]_{\kappa}+\left[\mathcal{J} \frac{\partial \xi_{l}}{\partial x_{m}}\right]_{\kappa} \mathrm{D}_{\xi_{l}}\right),
$$

where $J_{\kappa}^{-1}$ is the determinant of the Jacobian, whereas $\left[\mathcal{J} \frac{\partial \xi_{l}}{\partial x_{m}}\right]$ represent the metric terms. These terms appear in the discretization of the PDEs in curvilinear coordinates and have to satisfy discrete geometric conservation law constraints. We computed these metric terms as described in Reyna Nolasco et al. [43] for conforming interfaces. It is then possible to integrate the system of ordinary differential equations (2) in time with, for example, an $s$ stage (explicit or implicit) Runge-Kutta method. Herein, the ERK schemes are optimized using of the spectrum of the operator used to discretize the two-dimensional advection equation.

\section{B. Spectrum of the two-dimensional advection equation}

The linear convection equation in Cartesian physical coordinates is written as

$$
\frac{\partial \mathcal{U}}{\partial t}+\sum_{m=1}^{2} \frac{\partial\left(a_{m} \mathcal{U}\right)}{\partial x_{m}}=0
$$

where $\left(a_{m} \mathcal{U}\right)$ and $a_{m}$ denote the inviscid fluxes and the (constant) components of the convection speed, respectively, while $a_{m}$ satisfies $\sqrt{a_{1}^{2}+a_{2}^{2}}=1$. The computational domain is $x_{1} \in[0,1]$, and $x_{2} \in[0,1]$. On all the boundaries of the spatial domain, we imposed periodic boundary conditions. The spatial discretizations referred to in the previous section are used to discretize the advection operator. By varying the velocity vector angle in the range $\left[0,90^{\circ}\right]$, we compute multiple spectra of the global spatial discretization matrix. Approximately $1.44 \times 10^{4} \mathrm{DOFs}$, for all the order of accuracy was used. Elements with an aspect ratio of one (i.e., squares) comprise the grid. The numerical technique 
that used to optimize explicit Runge-Kutta schemes given a set of eigenvalues of spatial discretization is described in the next section.

\section{Design of the optimal explicit Runge-Kutta schemes}

In this section, new optimized explicit Runge-Kutta (ERK) schemes are developed for linear wave propagation problems discretized with entropy stable collocated discontinuous Galerkin methods with the SBP property. Based on the method of line approach, the spatial discretization of time-dependent partial differential equations leads to an initial value problem of the form

$$
G^{\prime}(t)=F(G), \quad G(0)=G_{0},
$$

where $G(t): \mathbb{R} \rightarrow \mathbb{R}^{N^{\text {dof }}}$ and $F: \mathbb{R}^{N^{\text {dof }}} \rightarrow \mathbb{R}^{N^{\text {dof }}}$ are the vector of the unknowns and the vector of the residuals, respectively. The symbol $N^{\text {dof }}=N \times(p+1)^{3}$ denotes the number of degrees of freedom (DOFs), where $N$ is the number of cells used to discretize the domain, $\Omega$, whereas $p$ is the solution polynomial degree used in each cell. Note that only conforming interface elements are considered here, and therefore, $p$ is the same for all cells in the domain. The time discretization of the system (6) with an ERK scheme with $s$ stages yields the following time step iteration

$$
\begin{aligned}
y_{i} & =G^{n}+\Delta t \sum_{j=1}^{s} a_{i j} F\left(t_{n}+c_{j} \Delta t, y_{j}\right), \quad i=1, \ldots, s, \\
G^{n+1} & =G^{n}+\Delta t \sum_{i=1}^{s} b_{i} F\left(t_{n}+c_{i} \Delta t, y_{i}\right),
\end{aligned}
$$

where $y_{i}$ represents the $i$-th stage value of the ERK method. The composition of the coefficients matrix $A \in \mathbb{R}^{s \times s}$, the weights $b \in \mathbb{R}^{s}$, and the nodes $c \in \mathbb{R}^{s}$ gives the Butcher tableau [31]. We design new ERK schemes that maximize the stable time step size while minimizing the leading truncation error constant and achieving low storage requirements. The convex optimization technique and the bisection algorithm described in $[26,27]$ are the key elements of the optimization procedure we use here. These algorithms are implemented in RK-Opt [44], a package for the design of numerical ODE solvers.

\section{A. Stability polynomial}

Consider the following linear constant-coefficient initial value problem

$$
G^{\prime}(t)=\lambda G, \quad G(0)=G^{0},
$$

where $\lambda \in \mathbb{C}$. By using a Runge-Kutta scheme to integrate in time Equation (8), we have the following iteration

$$
G^{n+1}=R(\Delta t \lambda) G^{n}
$$

where $R=R(\Delta t \lambda)=R(z)$ denotes the stability function and the argument $\Delta t$ is the (time) step size. The expression of $R(z)$ depends on the coefficients of the Runge-Kutta method [31, 45]. For an $s$-stage ERK scheme, the stability 
function is a polynomial of degree $s$ in the variable $z$,

$$
R(z)=\sum_{j=0}^{s} \beta_{j} z^{j}
$$

At each time step, the solution can be obtained by (9), as the stability of the method depends on $|R(z)|[31]$. Then, the absolute stability region, $S$, of the scheme is given by

$$
S=\{z \in \mathbb{C}:|R(z)| \leq 1\}, \text { where } z \equiv \Delta t \lambda \in S
$$

It is clearly demonstrated (see, for instance, [26, 27]) that when an ERK time integration scheme is applied to a linear constant coefficients system of ODEs, such as the one obtained by applying the aforementioned entropy stable SBP-SAT spatial operator to the advection equation, the following iteration is given

$$
G^{n+1}=R\left(\chi \lambda_{i}\right) G^{n}, \quad \text { for } i=1, \ldots, N^{\text {dof }}
$$

In Equation (12), the symbol $\chi$ represents the Courant-Friedrichs-Lewy (CFL) number, whereas the $\lambda_{i}, i=1, \ldots, N^{\text {dof }}$ form the spectrum of the discrete spatial operator. Therefore, the solution of the ODE system is stable if $\left(\chi \lambda_{i}\right) \in S$ for $1 \leq i \leq N^{\text {dof }}$. Given a mesh and a spatial discretization, the maximum absolutely stable CFL number (and hence step size) should satisfy

$$
\chi_{\text {stab }}=\max \left\{\chi \geq 0:\left|R\left(\chi \lambda_{i}\right)\right| \leq 1, \text { for } i=1, \ldots, N^{\mathrm{dof}}\right\}
$$

For a $p$ th order ERK, the first $p+1$ terms $(0 \leq j \leq p)$ of the stability polynomial (10) must be identical to the exponential function, which is the exact solution of Equation (8) [31]. Thus, $R(z)$ is expressed as follows,

$$
\mathrm{R}(z)=\sum_{j=0}^{p} \frac{1}{j !} z^{j}+\sum_{j=p+1}^{s} \beta_{j} z^{j}
$$

Consequently, the optimal stability polynomials can be obtained by selecting the coefficients $\left[\beta_{j}\right]_{j=p+1}^{j=s}$ in (14) that maximize $\chi_{\text {stab }}$. Therefore, given the ODE system (6), the optimization problem for the ERK schemes can be stated as

Problem 1 Given the spectrum of the Jacobian of $F(G)$, i.e., $\sigma=\left\{\lambda_{i}, i=1, \ldots, N^{d o f}\right\}$,

$$
\begin{array}{cl}
\underset{\beta_{p+1}, \ldots, \beta_{s}}{\operatorname{maximize}} & \chi \\
\text { subject to } & \left|\mathrm{R}\left(\chi \lambda_{i}\right)\right| \leq 1 \text { for all } \lambda_{i}, \\
& \mathrm{R}(z)-\exp (z)=O\left(z^{p+1}\right) .
\end{array}
$$

The convex optimization technique and bisection algorithm described in $[26,27]$ with respect to the CFL number, $\chi$, are applied to optimize the coefficients of the ERK method. Specifically, we fix $\chi$ and solve the resulting convex feasibility problem to determine whether there exists a choice of coefficients $\beta$ that satisfy the constraints. The upper bound for the initial bisection interval is $10 \mathrm{~s}$, and we use a monomial basis. The bisection search is carried out to an accuracy of $10^{-7}$. This technique allows us to optimize methods with large numbers of stages in order to improve the maximum absolutely stable time step, $\chi_{\text {stab }}$. 
The optimization is carried out for second- to fifth-order accurate schemes, where the order in time matches the order in space. The set of eigenvalues used for the optimization process is obtained by the union of the spectra of test problem (see Section II.B). The stability region and the scaled set of eigenvalues $(\Delta t \lambda)$ of fifth-order accurate methods for the advection equation is shown in Figure 1. Specifically, the fifth-order optimized scheme with sixteen stages and the traditional Runge-Kutta-Fehlberg six-stage fifth-order accurate method are plotted. It is evident that all the scaled eigenvalues $(\Delta t \lambda)$ fall inside the stability regions. In Figure 1b, we also observe that a larger step size can be used with the optimized explicit Runge-Kutta schemes. However, as the Section III.C shows, the method's efficiency should be measured relatively to the number of stages $s$ (i.e., considering the effective time step).

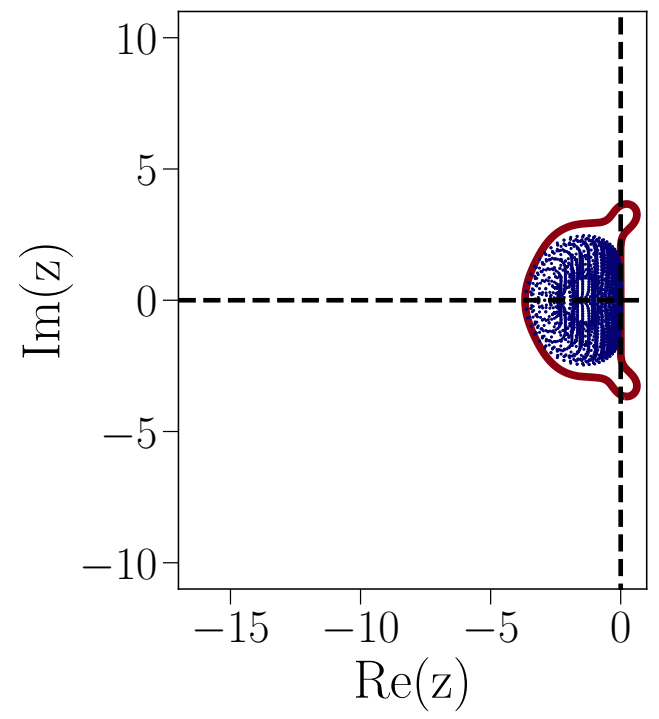

(a) $\operatorname{ERKF}(6,5)$

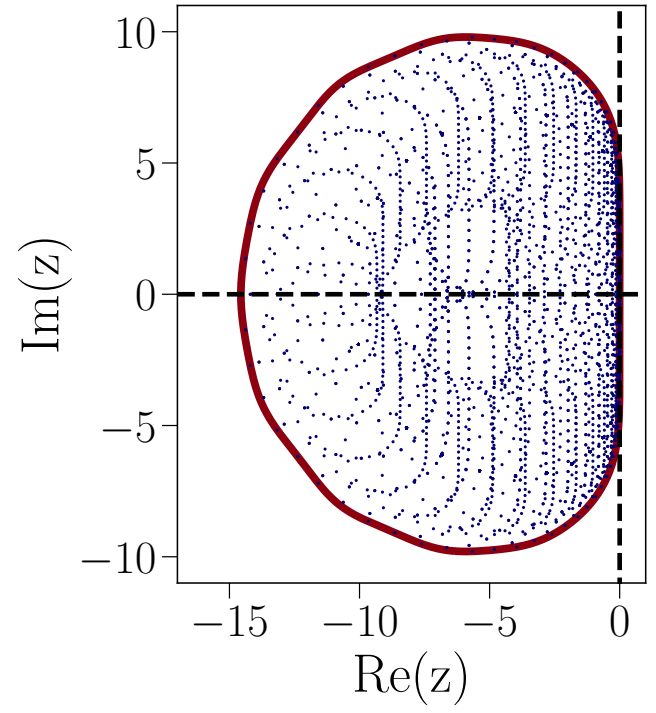

(b) $\operatorname{ERK}(16,5)$

Figure 1 Stability region (red line) and scaled spectrum (blue dots) for the advection equation.

\section{B. Coefficients of the Runge-Kutta schemes}

The stability polynomial computation is the first step of the numerical technique to design the optimal ERK methods. The selection of stability polynomial does not fully determine the method. The ERK method with $s$ stages has $s(s+1) / 2$ coefficients and only $s$ of them are constrained by the stability polynomial. The second step is therefore the computation of the Butcher coefficients $A, b$, and $c$ of the schemes $[31,45]$ that correspond to the stability polynomial coefficients described in the previous section. Additional nonlinear order conditions can be satisfied using the remaining degrees of freedom [31] to obtain a low-storage implementation, and ensure that the truncation error coefficients are not too large. The stability polynomial determines the linear accuracy of the method, whereas the nonlinear accuracy depends on a larger set of order conditions $\tau_{i}^{(r)}(\mathbf{A}, \mathbf{b})=0$, where $0 \leq r \leq p$ [31]. We ensure that those conditions are satisfied up to order $p$. Here, we use a low-storage form with three registers per stage [46] that aims at reducing the memory footprint when a large number of stages, $s$, is used (see Algorithm 1). The storage conditions are imposed on the scheme to be written in the minimum-storage form, which is given by $\Gamma(\mathbf{A}, \mathbf{b})=0$. Finally, the Euclidean norm of the truncation error coefficients of order $p+1$,

$$
C^{(p+1)}=\left(\sum_{i} \tau_{i}^{(p+1)}\right)^{1 / 2},
$$




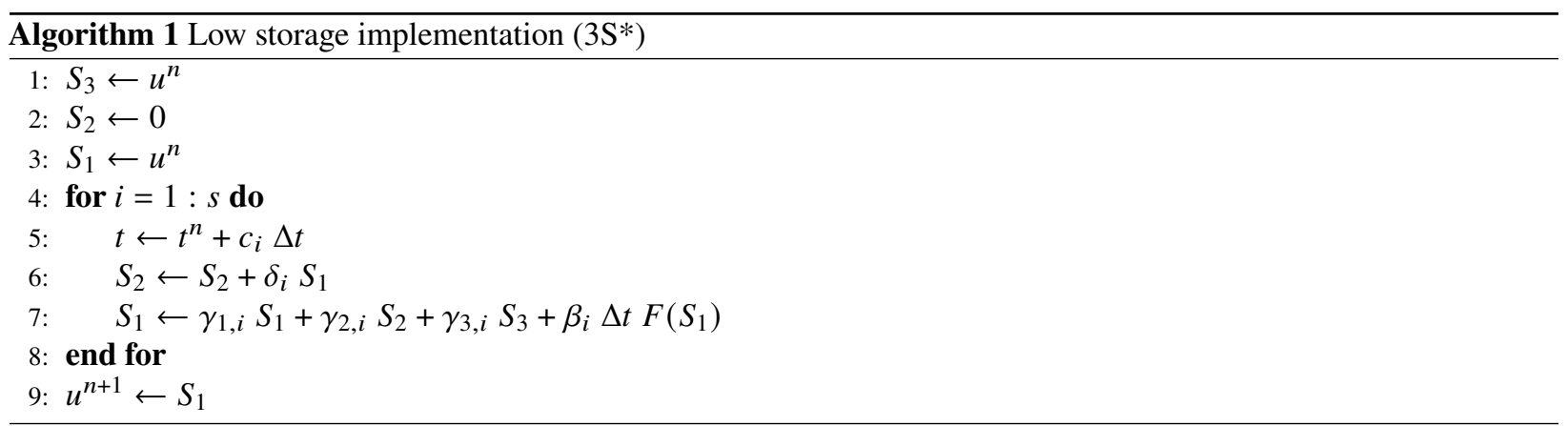

is also minimized [31, 45]. In summary, the optimization problem reads [27]

Problem 2 Given the optimal stability polynomial coefficients $\beta_{j}$

$$
\begin{array}{cl}
\underset{A, b}{\operatorname{minimize}} & C^{(p+1)} \\
\text { subject to } & \tau_{i}^{(r)}(\mathbf{A}, \mathbf{b})=0,(0 \leq r \leq p), \\
& \mathbf{b}^{T} \mathbf{A}^{j-1} \mathbf{e}=\beta_{j},(0 \leq j \leq s), \\
& \Gamma(\mathbf{A}, \mathbf{b})=0 .
\end{array}
$$

To solve problem (2), we used RK-Opt, a package for the design of numerical ODE solvers [47].

\section{Efficiency and CFL number}

The relative efficiency of two ERK methods of order $p$ can be measured by $\zeta_{s t a b}$ (or $\zeta_{a c c}$ ), if stability (or accuracy) is the more restrictive concern. The expression of $\zeta_{\text {stab }}$ and $\zeta_{a c c}$ are given by

$$
\zeta_{s t a b}=\frac{\sigma \chi_{1} / s_{1}}{\sigma \chi_{2} / s_{2}}=\frac{\chi_{1} / s_{1}}{\chi_{2} / s_{2}}, \quad \zeta_{a c c}=\left(\frac{C_{2}^{(p+1)}}{C_{1}^{(p+1)}}\right)^{\frac{1}{p}} \frac{s_{2}}{s_{1}},
$$

where $\chi_{1,2}$ and $C_{1,2}^{(p+1)}$ denote the maximum effective stable CFL number and the principal error norms of the two ERK schemes, respectively. The symbol $\sigma$ represents a safety factor applied to both schemes. Figure 2 shows $\zeta_{s t a b}$ and $\zeta_{a c c}$ for second- to fifth-order accurate optimized ERK schemes versus the "traditional" ERK schemes [31]. The "traditional" ERK methods considered here are the mid-point rule two-stage second-order accurate (ERK $(2,2))$ scheme, the Heun's three-stage third-order accurate $(\operatorname{ERK}(3,3))$ method, the classical four-stage fourth-order accurate $(\operatorname{ERK}(4,4))$ scheme, and the Runge-Kutta-Fehlberg six-stage fifth-order accurate $(\operatorname{ERKF}(6,5))$ method. In Figure 2 the new schemes are optimized using the eigenvalues of the discrete spatial operator of the advection equation. The maximum stable CFL number $\chi_{s t a b}$ is also plotted in Figure 2, and indicated by the green dots curve.

The blue dots curve and the red dots curve indicate the stability efficiency, $\zeta_{s t a b}$, and the accuracy efficiency, $\zeta_{a c c}$. We demonstrate that, due to the poor numerical conditioning of the problem for very large values of $s$, our optimized schemes are tailored for $s \leq 16$. There is a practical reason: for $s>16$ a degradation of the performance of the optimization toolbox is observed, which results in unreliable schemes. This is shown in Figure 2 and in the table included in the Appendix VI, where sudden changes in performance or an oscillatory behavior is noticed. However, it is necessary to note that adding more stages does not cause a significant improvement in efficiency. The asymptotic gain that may be achieved by adding stages is indeed bounded, as the classical CFL condition implies that the scheme 


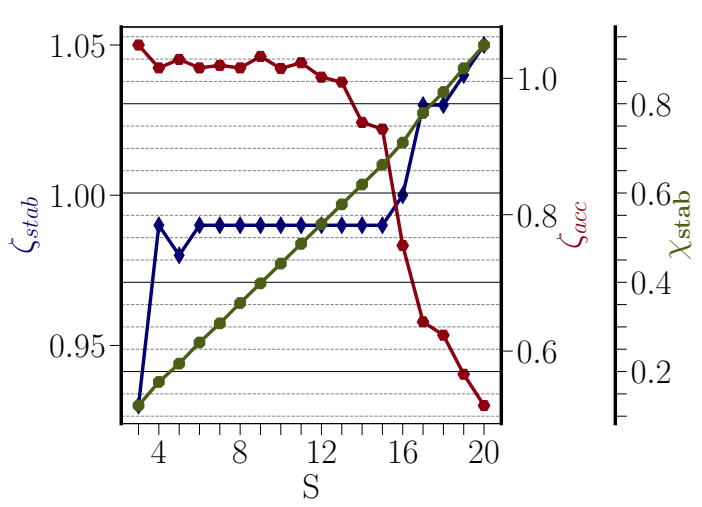

(a) $2^{\text {nd }}$ optimized ERK vs. ERK(2,2) scheme.

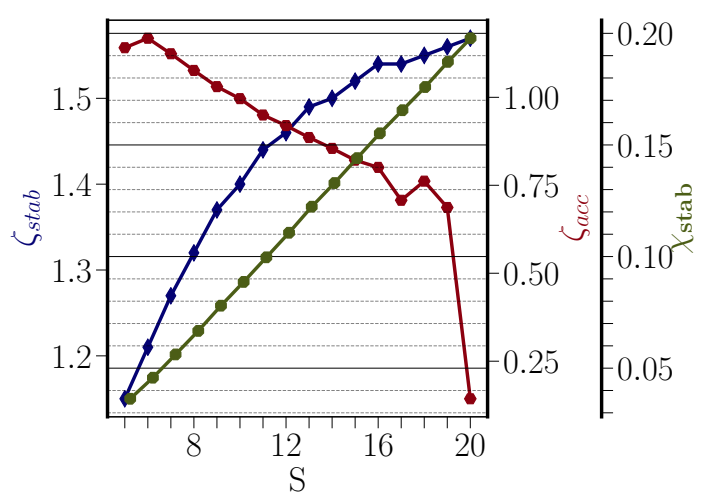

(c) $4^{\text {th }}$ optimized ERK vs. ERK $(4,4)$ scheme.

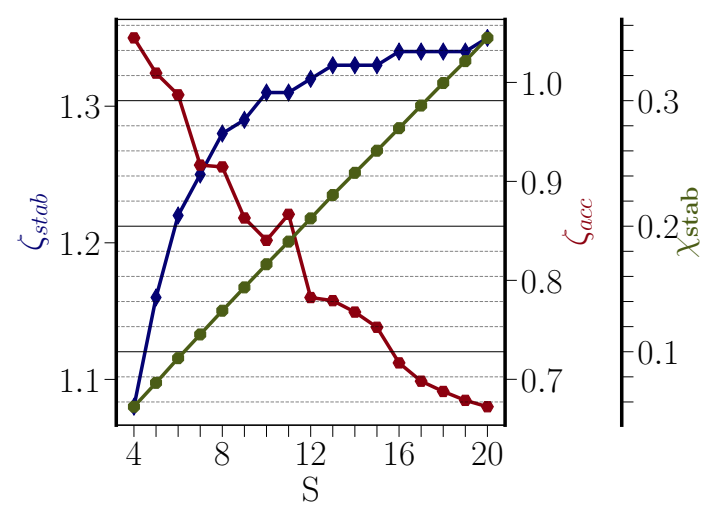

(b) $3^{\text {rd }}$ optimized ERK methods vs. ERK $(3,3)$ scheme.

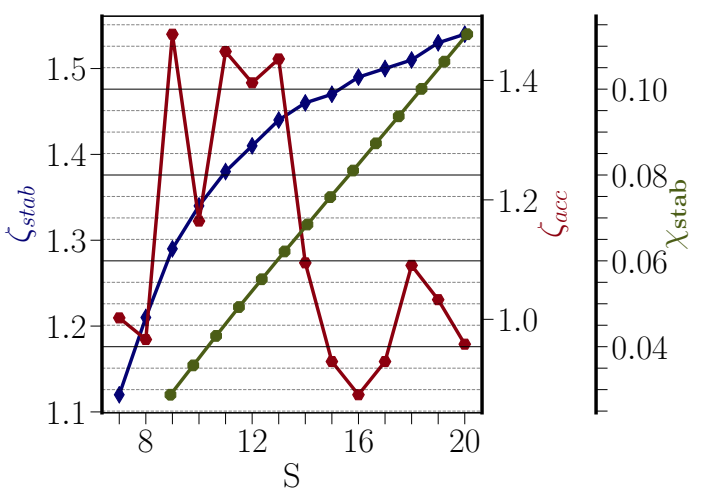

(d) $5^{\text {th }}$ optimized ERK methods vs. $\operatorname{ERKF}(6,5)$ scheme.

Figure 2 Efficiencies and maximum linearly stable CFL number.

cannot be stable for a CFL number greater than $s$ [48]. Furthermore, we can see that the global error efficiency of the optimized schemes decreases as the number of stages increases for the optimized methods. The low-storage coefficients and the values of $\zeta_{s t a b}$ and $\zeta_{a c c}$ of the optimized ERK methods are included in the Appendix VI.

\section{Performance of the optimized schemes}

For the efficiency and accuracy of the optimized ERK schemes to be assessed, a convergence study is shown in this section for the advection equation and the propagation of an isentropic vortex with the compressible Euler equations. The analysis is provided for reference and some optimized ERK methods.

\section{A. Convergence study}

Here we present the optimized ERK scheme convergence study for some selected classic and optimized ERK methods. For this study to be carried out, a system of nonlinear non-autonomous system of ODE is implemented, as 
proposed by Stanescu and Habashi [49] and Niegemann et al. [50],

$$
\begin{aligned}
& \frac{d q_{1}}{d t}=\frac{1}{q_{1}}-q_{2} \frac{e^{t^{2}}}{t^{2}}-t \\
& \frac{d q_{2}}{d t}=\frac{1}{q_{2}}-e^{t^{2}}-2 t e^{-t^{2}}
\end{aligned}
$$

with the following initial conditions: $q_{1}\left(t^{0}\right)=1$, and $q_{2}\left(t^{0}\right)=e^{-1}$. The time interval is $t \in\left[t^{0}=0, t^{e}=1.4\right]$, and the analytical solution given as $q_{1}(t)=1 / t$, and $q_{2}(t)=e^{-t^{2}}$. The error modulus is used in this study and is defined as

$$
\varepsilon\left(t^{e}\right)=\left(Q_{1}\left(t^{e}\right)-q_{1}\left(t^{e}\right)\right)+\left(Q_{2}\left(t^{e}\right)-q_{2}\left(t^{e}\right)\right)
$$

where $Q_{1}$ and $Q_{2}$ represent the numerical solutions. Figure 3 demonstrates that all the schemes attain the theoretical convergence rate, and the new optimized methods display notably smaller errors than the reference schemes.

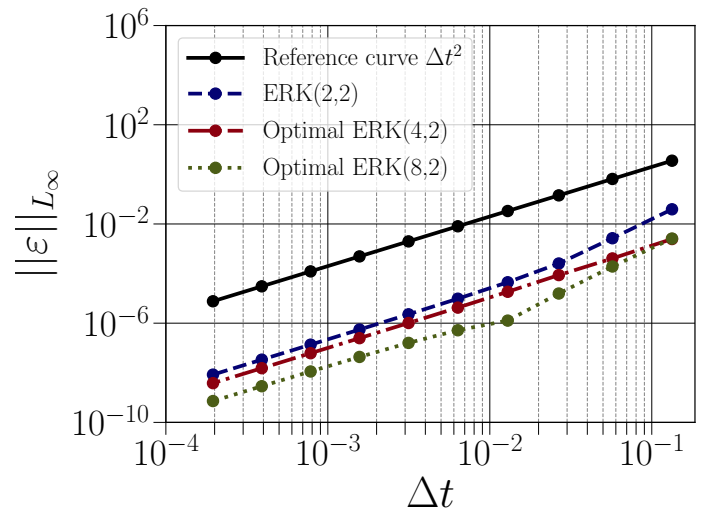

(a) $2^{\text {nd }}$ ERK schemes.

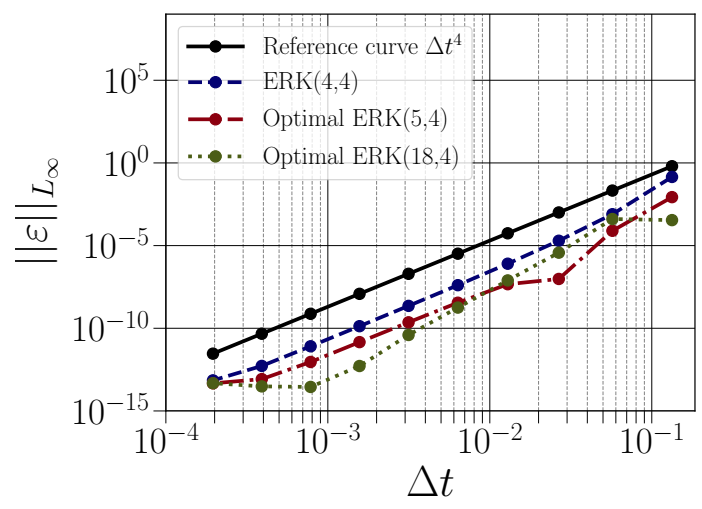

(c) $4^{\text {th }}$ ERK schemes.

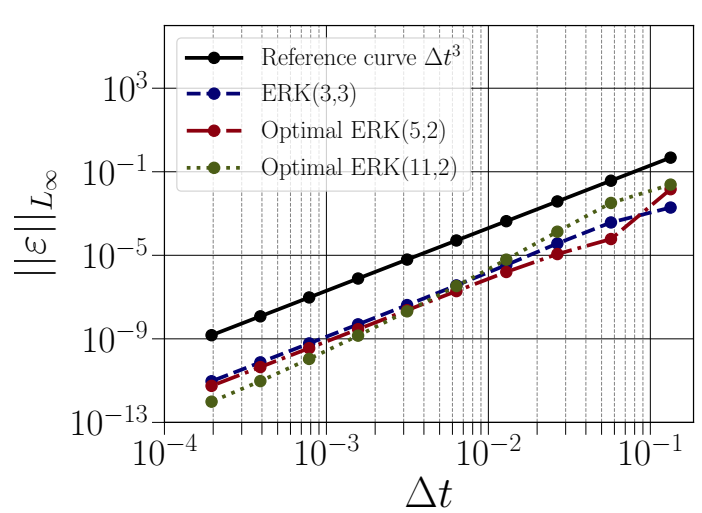

(b) $3^{\text {rd }}$ ERK schemes.

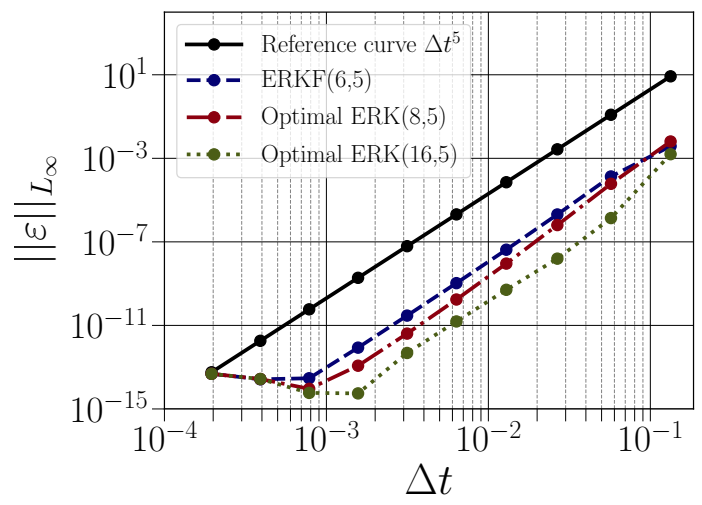

(d) $5^{\text {th }}$ ERK schemes.

Figure 3 Convergence study of the optimized ERK methods. 


\section{B. Error and computational cost}

The performance of some numerical simulations is required in order to assess the superior efficiency and accuracy of the new optimized schemes compared to the classic ERK methods selected. The advection equation and the propagation of the isentropic vortex are used here as numerical simulation test cases. The maximum norm of the error is reported. A time interval $\left[t^{0}=0, t^{e}=2\right]$ is applied to all the simulations, and a constant number of $4 \times 10^{4}$ DOFs for all the orders of accuracy is used. The angle that the convective velocity vector forms with the horizontal axis equal to $45^{\circ}$ sets in the advection equation simulation, where the computational domain is the same as this described in II.B. For the second test case, the compressible Euler equations in Cartesian coordinates read

$$
\frac{\partial \boldsymbol{q}}{\partial t}+\sum_{m=1}^{3} \frac{\partial \boldsymbol{F}_{x_{m}}}{\partial x_{m}}=0,
$$

where $\boldsymbol{q}$ are the conserved variables and $\boldsymbol{F}_{x_{m}}$ are the inviscid fluxes. The exact solution of the isentropic vortex test case is given as

$$
\left\{\begin{array}{l}
\mathcal{G}\left(x_{1}, x_{2}, x_{3}, t\right)=1-\left\{\left[\left(x_{1}-x_{1,0}\right)-\mathcal{U}_{\infty} \cos (\alpha) t\right]^{2}+\left[\left(x_{2}-x_{2,0}\right)-\mathcal{U}_{\infty} \sin (\alpha) t\right]^{2}\right\} \\
\rho=T^{\frac{1}{\gamma-1}} \\
\mathcal{U}_{1}=\mathcal{U}_{\infty} \cos (\alpha)-\epsilon_{v} \frac{\left(x_{2}-x_{2,0}\right)-\mathcal{U}_{\infty} \sin (\alpha) t}{2 \pi} \exp \left(\frac{\mathcal{G}}{2}\right), \\
\mathcal{U}_{2}=\mathcal{U}_{\infty} \sin (\alpha)-\epsilon_{v} \frac{\left(x_{1}-x_{1,0}\right)-\mathcal{U}_{\infty} \cos (\alpha) t}{2 \pi} \exp \left(\frac{\mathcal{G}}{2}\right), \\
\mathcal{U}_{3}=0 \\
T=\left[1-\epsilon_{\nu}^{2} \operatorname{Ma}_{\infty}^{2} \frac{\gamma-1}{8 \pi^{2}} \exp (\mathcal{G})\right] .
\end{array}\right.
$$

where $\mathcal{U}_{\infty}, \mathrm{Ma}_{\infty}$, and $\left(x_{1,0}, x_{2,0}, x_{3,0}\right)$ denote the modulus of the free-stream velocity, the free-stream Mach number, and the vortex center, respectively, where following values are fixed: $\mathcal{U}_{\infty}=\mathrm{Ma}_{\infty} c_{\infty}, \epsilon_{v}=5, \gamma=1.4$, and $\left(x_{1,0}, x_{2,0}, x_{3,0}\right)=(0,0,0)$. The computational domain is $x_{1} \in[-5,5], x_{2} \in[-5,5]$, and $x_{3} \in[-5,5]$. The angle of the vortex propagation and the background flow Mach number are set to $\alpha=45^{\circ}$, and $\mathrm{Ma}_{\infty}=0.5$, respectively. The step size control is applied for all simulations here, and takes the form

$$
\Delta t_{n}=\sigma \mathrm{CFL} \min _{i} \frac{\Delta x_{i}}{v_{i}},
$$

CFL is the desired CFL number, $\Delta x_{i}$ represents a mesh-spacing local measurement, and $v_{i}$ represents the maximum local wave speed measurement, in relation to the largest eigenvalue of the flux Jacobian (in magnitude). The space and time discretization details are the factors that need to be considered to select the optimal CFL; we used $\chi_{\text {stab }}$ in this work, which is the maximum CFL number reached during the procedure of optimization (see Section III.C). The local mesh spacing ratio and the maximal speed at a node $i$ is estimated in this case as

$$
\frac{\Delta x_{i}}{v_{i}}=\gamma \frac{J_{i}}{\sum_{j=1}^{d}\left|\left(J \partial_{x} \xi^{j}\right)_{i} \cdot \tilde{v}_{i}\right|},
$$

where $d$ is the number of spatial dimensions, $\tilde{v}_{i}$ is the velocity, $J_{i}$ the determinant of the grid Jacobian $\partial_{x} \xi$ at node $i,\left(J \partial_{x} \xi^{j}\right)_{i}$ is the contravariant basis vector in direction $j$ at node $i$ [51], and $\gamma$ is a normalizing factor depending on the polynomial degree $p$ which is selected so that a real stability interval of 2, which is characteristic of the Heun's method [52], corresponds to CFL $=1$. A safety factor of $\sigma=0.95$ is used for all the simulations in (21). Tables 1 


\begin{tabular}{l|l|l|l}
\hline \hline ERK Schemes & $L_{\infty}$ & Wall-clock time [s] & Function evaluations \\
\hline$(2,2)$ & $3.4145 \mathrm{e}-02$ & 1.5046 & 596.0 \\
$(4,2)$ & $3.4145 \mathrm{e}-02$ & 1.4351 & 596.0 \\
$(8,2)$ & $3.4333 \mathrm{e}-02$ & 1.4257 & 600.0 \\
$(3,3)$ & $3.8940 \mathrm{e}-04$ & 2.4771 & 1272.0 \\
$(5,3)$ & $4.8652 \mathrm{e}-04$ & 2.1954 & 1155.0 \\
$(11,3)$ & $9.3474 \mathrm{e}-04$ & 1.9439 & 1023.0 \\
$(4,4)$ & $2.5376 \mathrm{e}-05$ & 4.1705 & 2064.0 \\
$(6,4)$ & $2.5838 \mathrm{e}-05$ & 3.4634 & 1728.0 \\
$(15,4)$ & $5.8703 \mathrm{e}-05$ & 2.774 & 1365.0 \\
$(6,5)$ & $1.5259 \mathrm{e}-06$ & 5.7688 & 2862.0 \\
$(8,5)$ & $1.5188 \mathrm{e}-06$ & 4.7388 & 2360.0 \\
\hline \hline
\end{tabular}

Table 1 The $L_{\infty}$ norm of the error, wall-clock time in seconds, number of function evaluations of each schemes for the advection equation.

\begin{tabular}{l|l|l|l}
\hline \hline ERK Schemes & \multicolumn{1}{|c|}{$L_{\infty}$} & Wall-clock time [s] & Function evaluations \\
\hline$(2,2)$ & $3.4409 \mathrm{e}-03$ & 2.293 & 302.0 \\
$(4,2)$ & $3.4361 \mathrm{e}-03$ & 2.1937 & 292.0 \\
$(8,2)$ & $3.4360 \mathrm{e}-03$ & 2.2715 & 296.0 \\
$(3,3)$ & $1.4068 \mathrm{e}-04$ & 3.9003 & 645.0 \\
$(5,3)$ & $1.4082 \mathrm{e}-04$ & 3.4274 & 565.0 \\
$(11,3)$ & $1.4141 \mathrm{e}-04$ & 3.1176 & 495.0 \\
$(4,4)$ & $1.1954 \mathrm{e}-05$ & 6.4908 & 1052.0 \\
$(6,4)$ & $1.1954 \mathrm{e}-05$ & 5.1901 & 840.0 \\
$(15,4)$ & $1.1953 \mathrm{e}-05$ & 4.4329 & 660.0 \\
$(6,5)$ & $1.3277 \mathrm{e}-06$ & 9.6865 & 1464.0 \\
$(8,5)$ & $1.3285 \mathrm{e}-06$ & 6.7479 & 1000.0 \\
\hline \hline
\end{tabular}

Table 2 The $L_{\infty}$ norm of the error, wall-clock time in seconds, number of function evaluations of each schemes for the isentropic vortex propagation.

and 2 show that, as the order of the discretization increases, the error decreases. In contrast, the number of function evaluations increases for higher-order accurate methods, so the wall-clock time also increases. Therefore, the most efficient temporal integration scheme depends on the error tolerance targeted. The optimized schemes were clearly observed to increase the speed of the simulations over the standard methods. Specifically, the new fourth- and fifth-order methods with fifteen and sixteen stages respectively, provide a speed-up of approximately $35 \%$ for both the advection equation and the isentropic vortex propagation. It is notable that the optimized schemes also yield a decrease in the error of approximately one order of magnitude. 


\section{Application to viscous flows}

\section{A. Taylor-Green vortex problem}

In this section, we validate the efficiency and accuracy of the new temporal schemes for the compressible NavierStokes equations by simulating the three-dimensional Taylor-Green vortex (TGV) problem at a Reynolds number of $\operatorname{Re}=1.6 \times 10^{3}$, and Mach number $\mathrm{Ma}=0.1$. The initial condition is obtained by

$$
\begin{cases}\rho & =\rho_{0}+\frac{\gamma M_{a}^{2}}{16}\left[\cos \left(2 x_{1}\right)+\cos \left(2 x_{2}\right)\right]\left[\cos \left(2 x_{3}\right)+2\right] \\ \mathcal{U}_{1} & =\sin \left(x_{1}\right) \cos \left(x_{2}\right) \cos \left(x_{3}\right) \\ \mathcal{U}_{2} & =-\cos \left(x_{1}\right) \sin \left(x_{2}\right) \cos \left(x_{3}\right) \\ T & =T_{0}\end{cases}
$$

where $\rho, \mathcal{U}_{1}, \mathcal{U}_{2}$, and $T$ denote the density, temperature, and the velocity, respectively. The parameters for this test are $T_{0}=1, \rho_{0}=1$, and $\gamma=1.4$. Periodic conditions are enforced at all boundaries of the spatial computational domain. The number of DOFs is kept constant for all simulations and equal to $8 \times 10^{6}$, where the grids are equally spaced Cartesian grids with $100^{3}, 60^{3}, 50^{3}$, and $40^{3}$ number of elements for polynomial order $p=2,3,4$ and 5 , respectively. Simulations are carried out at a time interval from $t_{0}=0$ to $t_{f}=20$ time units. For second- to fourth-order accurate scheme simulations, we use a safety factor of $\sigma=0.95$. However, for fifth-order accurate schemes, it is harder to get a tight bound on the CFL scale factors. Thus, we use a safety factor of $\sigma=0.85$ for $5^{\text {th }}$ schemes. To analyze the accuracy of the new optimized schemes, the relative error in the kinetic energy dissipation rate is computed, where the predicted maximum stable CFL number is used, for the reference and the optimized ERK schemes. The evolution of the kinetic energy dissipation rate can be computed

$$
\epsilon_{E_{k}}(t)=-\frac{d E_{k}}{d t}
$$

where $E_{k}(t)$ is the temporal evolution of the integrated kinetic energy,and is obtained by

$$
E_{k}(t)=\frac{1}{\rho_{0} \Omega} \int_{\Omega} \rho \frac{\mathcal{U} \cdot \mathcal{U}}{2} d \Omega
$$

Figures 4 and 5 show the evolution of the integrated kinetic energy and the kinetic energy dissipation, respectively, for reference and the optimized ERK schemes. All the simulations are stable at their respective predicted CFL numbers. $E_{k}$ predictions are almost identical for the different schemes, while $\epsilon_{E_{k}}$ predictions improve with increase of grid density.

The analytical solution is not available for this test problem. Therefore, we numerically compute the reference solution by solving the problem with a constant number of $3.43 \times 10^{8}$ DOFs for all the order of accuracy. Figure 6 shows the relative error in the kinetic energy dissipation rate at the final time $t_{f}=20$ for the reference and the optimized methods. Clearly, both traditional and optimized high order accurate methods deliver a substantial improvement in accuracy.

In Figure 7, we measure the wall-clock time and the number of function evaluations for all simulations in order to verify the performance of the new temporal schemes. As we can see in Figure 7, by increasing the order of the accuracy, the new optimized schemes speedup the simulations compared to the traditional because they require fewer function evaluations. These results demonstrate that the performance of the new optimized schemes are much better than the traditional schemes in terms of the cost and the level of error. Specifically, the computational time saving for the $\operatorname{ERK}(4,2), \operatorname{ERK}(11,3), \operatorname{ERK}(15,4)$, and $\operatorname{ERK}(16,5)$ methods is $27 \%, 42 \%, 46 \%$, and $44 \%$, respectively. 


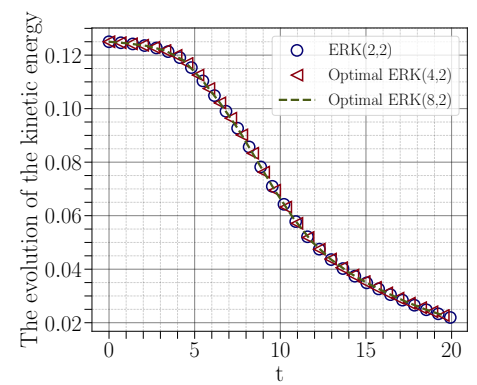

(a) Second-order ERK methods.

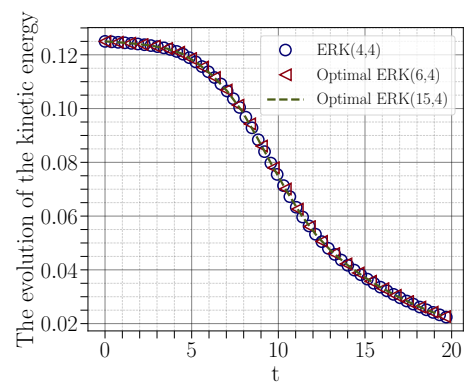

(c) Fourth-order ERK methods.

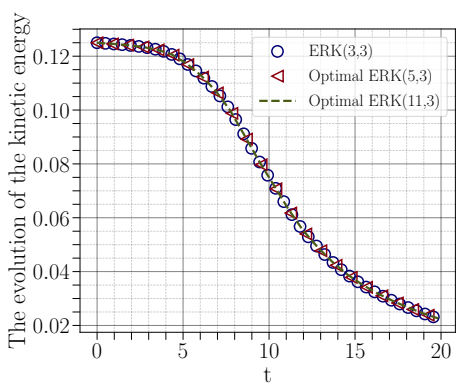

(b) Third-order ERK methods.

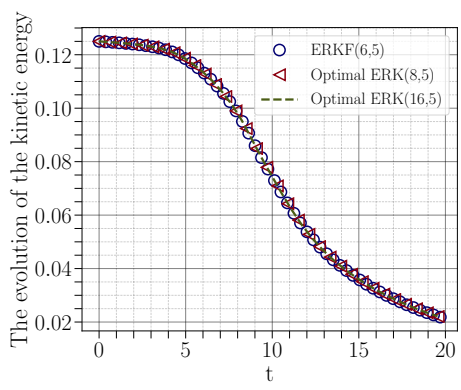

(d) Fifth-order ERK methods.

Figure 4 The evolution of the kinetic energy of the reference and optimized ERK methods for the TGV.

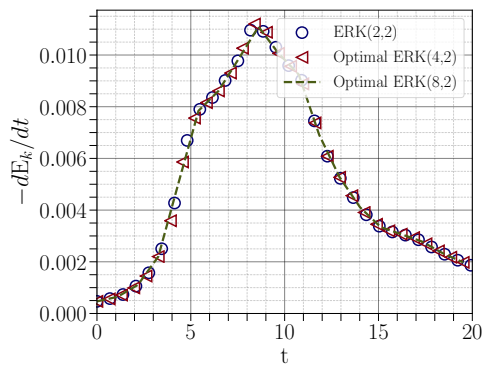

(a) Second-order ERK methods.

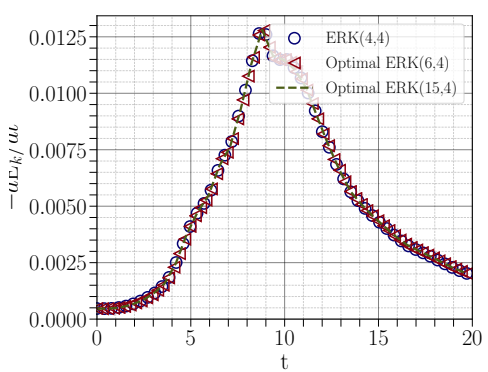

(c) Fourth-order ERK methods.

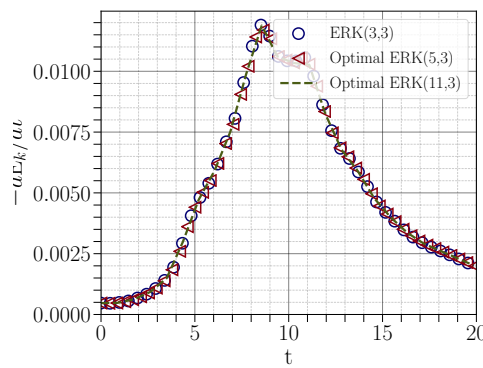

(b) Third-order ERK methods.

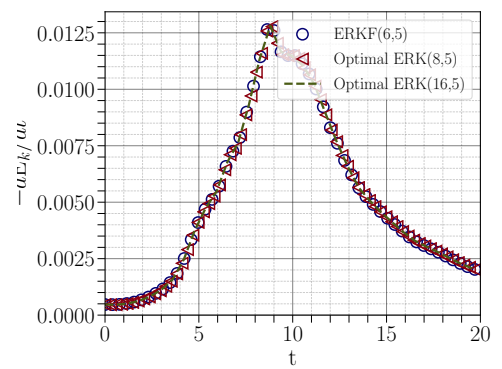

(d) Fifth-order ERK methods.

Figure 5 The evolution of the kinetic energy dissipation of the reference and optimized ERK methods for the TGV. 


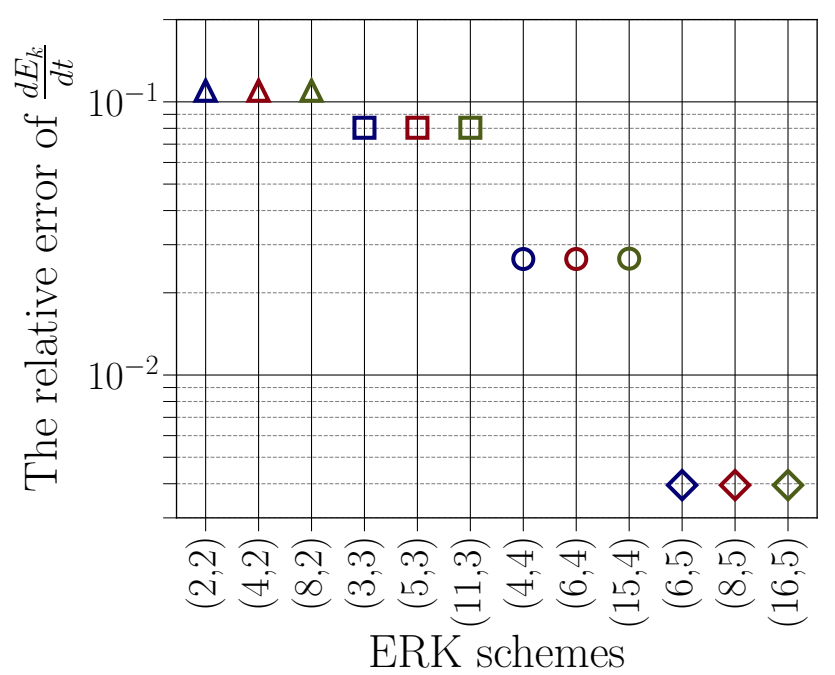

Figure 6 Relative error in kinetic energy dissipation rate for the reference and the optimized schemes.

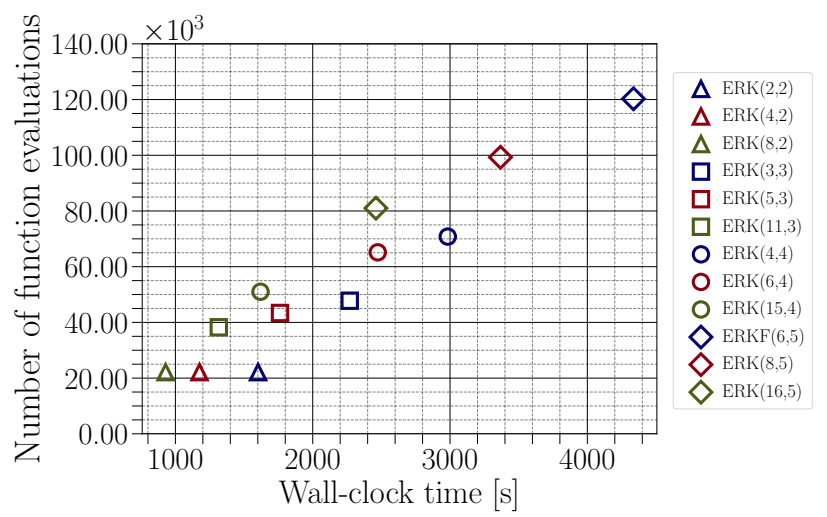

Figure 7 Wall-clock time in seconds, and number of function evaluations to simulate Taylor-green vortex test case.

\section{B. Flow past two identical spheres in tandem}

The flow past two identical spheres of diameter D is tested in this section. The spheres are located at separation distances of 10D. The testing for this problem was performed at a subcritical/transitional flow regime with a Reynolds number equal to $\operatorname{Re}=\mathcal{U}_{\infty} \mathrm{D} / v=3.9 \times 10^{3}$, and a near-incompressibility Mach number Ma $=0.1$. The upstream flow conditions of the first sphere are used to set the similarity parameters, e.g., $\mathcal{U}_{\infty}$ is the freestream velocity. Although the geometry is relatively straightforward, it remains quite difficult to capture the flow in this regime [24]. Thus, this is an interesting test case for assessing the accuracy, robustness, and efficiency of the new time integration schemes. We used one of the high-order grids in Gmsh format created by Steve Karman [53], to perform the numerical simulations. For our study, the grids comprise $\approx 1.9656 \times 10^{4}$ hexahedral cells. These spatial schemes remain constant for all the numerical simulations. The order of accuracy of the spatial discretization matches the order of accuracy of the time integration scheme in every simulation. If the degree of the solution and the number of cells in each block are given, we can determine the number of DOFs, which in this case is approximately $2.457 \times 10^{6}$. We imposed entropy stable adiabatic wall boundary conditions on the sphere surfaces. Far-field boundary conditions are applied to the remaining boundaries. For all the simulations, the safety factor is set to $\sigma=0.95$. This value of the safety factor is optimal because 
the schemes have been optimized by considering the spectra generated from uniform grids, and they have been applied in this test case that is highly unstructured grids. The contour plots are plotted using fifth-order accurate ERK time

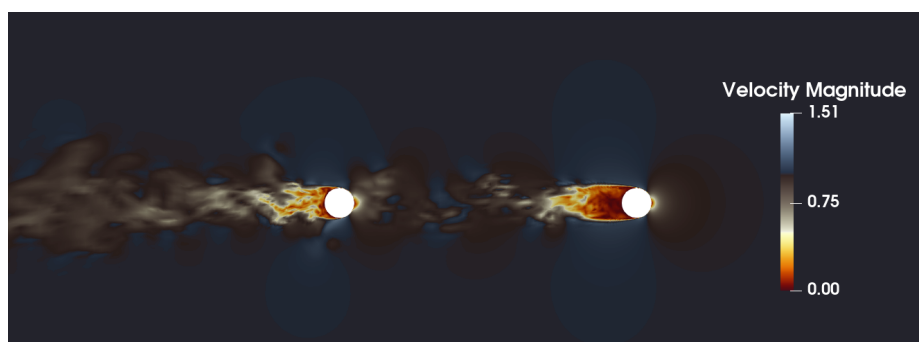

(a) $\operatorname{ERKF}(6,5)$

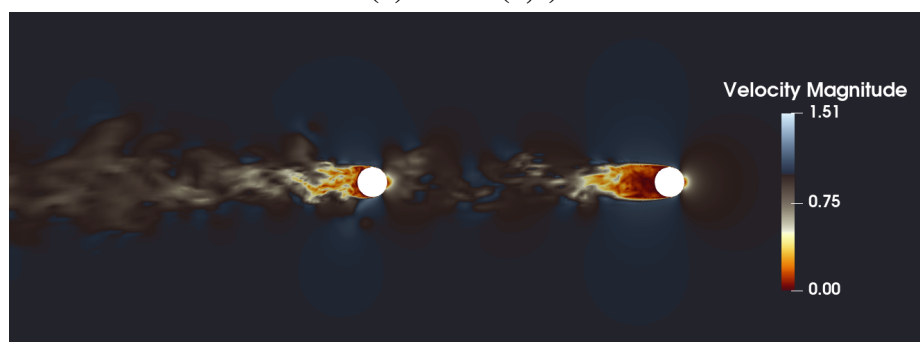

(b) $\operatorname{ERK}(8,5)$.

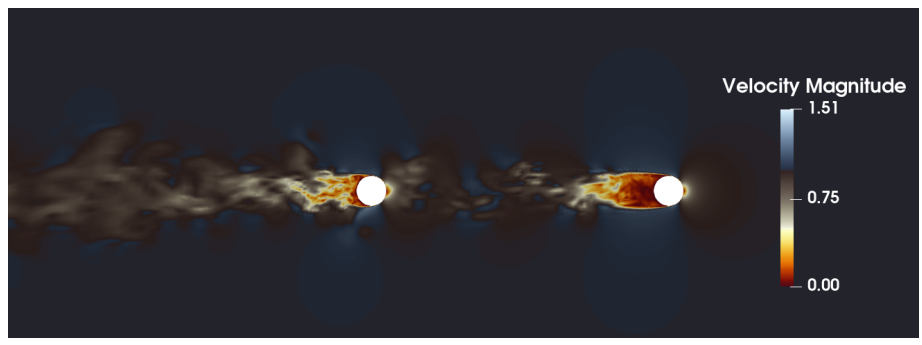

(c) $\operatorname{ERK}(16,5)$.

Figure 8 Contour plots of the velocity magnitude for the reference and the optimized schemes.

integration schemes in Figure 8. It is evident that similar patterns and flow features are obtained for all schemes. The reference $\operatorname{ERK}$ scheme (i.e., $\operatorname{ERKF}(6,5))$ and the new optimal schemes $\operatorname{ERK}(8,5)$ and $\operatorname{ERK}(16,5)$ do not display any obvious discrepancies, as shown in Figure 8. The wall-clock time was measured, and the number of function evaluations was counted for the reference and the optimized ERK schemes to verify the performance of the optimized schemes. The predicted maximum stable CFL number was used for each method. The results are shown in Figure 9. We can observe that by increasing the order of accuracy, the optimized schemes yield a greater speedup because they require fewer function evaluations. Therefore, the results shown in Figure 8 and Figure 9, lead us to conclude that the new schemes proposed can improve the overall solver performance. Specifically, the computational time saving for the ERK $(4,2)$, $\operatorname{ERK}(11,3), \operatorname{ERK}(15,4)$, and $\operatorname{ERK}(16,5)$ methods is $1 \%, 21 \%, 35 \%$, and $32 \%$, respectively. For the second-order schemes, the gain is positive; however, it is minimal. It is significant to note that the new temporal schemes, designed by linear advection equation on a uniform grid, achieves good results for a nonlinear system of PDEs and unstructured mesh. 


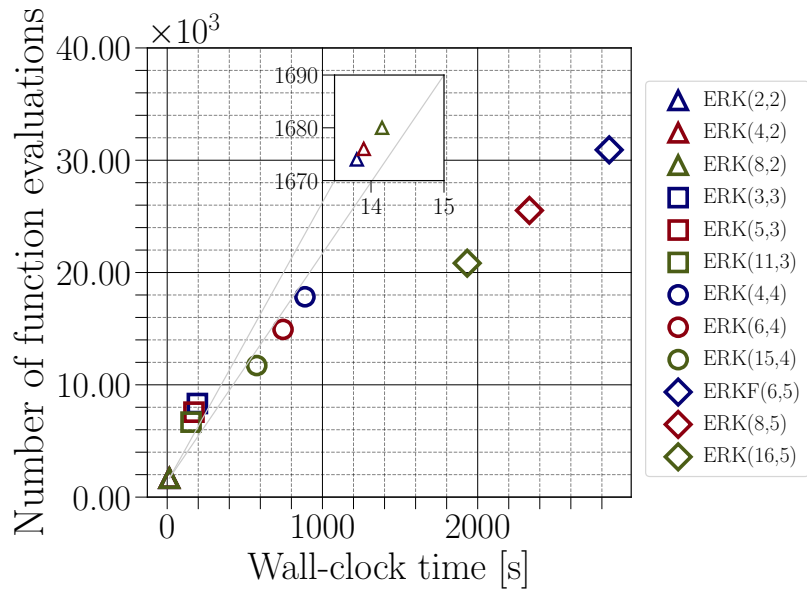

\section{Figure 9 Wall-clock time in seconds, and number of function evaluations to simulate flow past identical spheres in tandem.}

\section{Conclusions}

New explicit Runge-Kutta schemes optimized for entropy stable collocated discontinuous Galerkin methods have been proposed. The new schemes allow for a larger stable time step sizes and accurate solution, while admitting a low-storage implementation. To show the efficiency and the robustness of the new temporal schemes, the performance of the optimized methods for the three-dimensional Taylor-Green vortex problem at a Reynolds number of $\operatorname{Re}=1.6 \times 10^{3}$, and the flow past two identical spheres in tandem at a Reynolds number of $\operatorname{Re}=3.9 \times 10^{3}$ have been reported. The results indicate that the proposed schemes enhance the overall solver performance compared to widely used classical time integration schemes. 


\section{Appendix}

Efficiency and CFL number of the ERK schemes optimized using the eigenvalues of the advection equation

\begin{tabular}{c|c|c|c|c}
\hline \hline \multicolumn{2}{c|}{} & \multicolumn{2}{|c}{ Comparison with ERK(2,2) } \\
\hline & $\Delta t$ & $C^{(p+1)}$ & $\begin{array}{c}\zeta^{\text {stab }} \\
(\Delta t / s) \\
\text { ERK (2,2) }=4.4474 \mathrm{e}-02\end{array}$ & $\begin{array}{c}\zeta^{a c c} \\
(C)_{E R K(2,2)}^{(p+1)}=1.7180 \mathrm{e}-01\end{array}$ \\
\hline \hline 3 & $4.1292 \mathrm{e}-02$ & $6.9407 \mathrm{e}-02$ & 0.93 & 1.05 \\
4 & $4.4194 \mathrm{e}-02$ & $4.1667 \mathrm{e}-02$ & 0.99 & 1.02 \\
5 & $4.3537 \mathrm{e}-02$ & $2.6023 \mathrm{e}-02$ & 0.98 & 1.03 \\
6 & $4.4194 \mathrm{e}-02$ & $1.8519 \mathrm{e}-02$ & 0.99 & 1.02 \\
7 & $4.4003 \mathrm{e}-02$ & $1.3507 \mathrm{e}-02$ & 0.99 & 1.02 \\
8 & $4.4194 \mathrm{e}-02$ & $1.0417 \mathrm{e}-02$ & 0.99 & 1.02 \\
9 & $4.4185 \mathrm{e}-02$ & $7.9652 \mathrm{e}-03$ & 0.99 & 1.03 \\
10 & $4.4194 \mathrm{e}-02$ & $6.6776 \mathrm{e}-03$ & 0.99 & 1.01 \\
11 & $4.4190 \mathrm{e}-02$ & $5.4290 \mathrm{e}-03$ & 0.99 & 1.02 \\
12 & $4.4196 \mathrm{e}-02$ & $4.7559 \mathrm{e}-03$ & 0.99 & 1.00 \\
13 & $4.4194 \mathrm{e}-02$ & $4.1119 \mathrm{e}-03$ & 0.99 & 0.99 \\
14 & $4.4214 \mathrm{e}-02$ & $4.0072 \mathrm{e}-03$ & 0.99 & 0.94 \\
15 & $4.4216 \mathrm{e}-02$ & $3.5651 \mathrm{e}-03$ & 0.99 & 0.93 \\
16 & $4.4556 \mathrm{e}-02$ & $4.7107 \mathrm{e}-03$ & 1.00 & 0.75 \\
17 & $4.5834 \mathrm{e}-02$ & $5.7527 \mathrm{e}-03$ & 1.03 & 0.64 \\
18 & $4.5899 \mathrm{e}-02$ & $5.4584 \mathrm{e}-03$ & 1.03 & 0.62 \\
19 & $4.6286 \mathrm{e}-02$ & $5.9409 \mathrm{e}-03$ & 1.04 & 0.57 \\
20 & $4.6577 \mathrm{e}-02$ & $6.3495 \mathrm{e}-03$ & 1.05 & 0.52 \\
\hline \hline
\end{tabular}

Table 3 Stability, $\zeta^{(s t a b)}$, and accuracy, $\zeta^{(a c c)}$, efficiencies of the optimized second-order explicit Runge-Kutta methods for the advection equation.

\begin{tabular}{|c|c|c|c|c|}
\hline \multirow[b]{2}{*}{$s$} & \multirow[b]{2}{*}{$\Delta t$} & \multirow[b]{2}{*}{$C^{(p+1)}$} & \multicolumn{2}{|c|}{ Comparison with $\operatorname{ERK}(3,3)$} \\
\hline & & & $\begin{array}{c}\zeta^{\text {stab }} \\
(\Delta t / s)_{E R K(3,3)}=1.2981 \mathrm{e}-02\end{array}$ & $\begin{array}{c}\zeta^{a c c} \\
(C)_{E R K(3,3)}^{(p+1)}=4.6296 \mathrm{e}-02\end{array}$ \\
\hline 4 & $1.4049 \mathrm{e}-02$ & $1.7116 \mathrm{e}-02$ & 1.08 & 1.04 \\
\hline 5 & $1.5048 \mathrm{e}-02$ & $9.7231 \mathrm{e}-03$ & 1.16 & 1.01 \\
\hline 6 & $1.5813 \mathrm{e}-02$ & $6.0108 \mathrm{e}-03$ & 1.22 & 0.99 \\
\hline 7 & $1.6253 \mathrm{e}-02$ & $4.7343 \mathrm{e}-03$ & 1.25 & 0.92 \\
\hline 8 & $1.6586 \mathrm{e}-02$ & $3.1911 \mathrm{e}-03$ & 1.28 & 0.91 \\
\hline 9 & $1.6807 \mathrm{e}-02$ & $2.6676 \mathrm{e}-03$ & 1.29 & 0.86 \\
\hline 10 & $1.6970 \mathrm{e}-02$ & $2.1056 \mathrm{e}-03$ & 1.31 & 0.84 \\
\hline 11 & $1.7066 \mathrm{e}-02$ & $1.4424 \mathrm{e}-03$ & 1.31 & 0.87 \\
\hline 12 & $1.7185 \mathrm{e}-02$ & $1.5090 \mathrm{e}-03$ & 1.32 & 0.78 \\
\hline 13 & $1.7295 \mathrm{e}-02$ & $1.2013 \mathrm{e}-03$ & 1.33 & 0.78 \\
\hline 14 & $1.7323 \mathrm{e}-02$ & $1.0058 \mathrm{e}-03$ & 1.33 & 0.77 \\
\hline 15 & $1.7343 \mathrm{e}-02$ & $8.6886 \mathrm{e}-04$ & 1.34 & 0.75 \\
\hline 16 & $1.7380 \mathrm{e}-02$ & $8.2931 \mathrm{e}-04$ & 1.34 & 0.72 \\
\hline 17 & $1.7419 \mathrm{e}-02$ & $7.4780 \mathrm{e}-04$ & 1.34 & 0.70 \\
\hline 18 & $1.7448 \mathrm{e}-02$ & $6.5893 \mathrm{e}-04$ & 1.34 & 0.69 \\
\hline 19 & $1.7448 \mathrm{e}-02$ & $5.8280 \mathrm{e}-04$ & 1.34 & 0.68 \\
\hline 20 & $1.7500 \mathrm{e}-02$ & $5.1419 \mathrm{e}-04$ & 1.35 & 0.67 \\
\hline
\end{tabular}

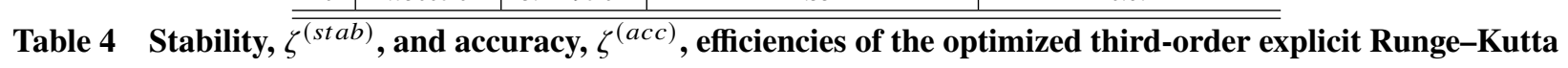
methods for the advection equation. 


\begin{tabular}{c|c|c|c|c}
\hline \hline & & \multicolumn{2}{|c}{ Comparison with ERK(4,4) } \\
\hline & $\Delta t$ & $C^{(p+1)}$ & $(\Delta t / s)_{E R K(4,4)}=6.3141 \mathrm{e}-03$ & $(C)_{E R K(4,4)}^{(p+1)} \zeta^{\text {stab }}$ \\
$s$ & $\Delta t .4505 \mathrm{e}-02$ \\
\hline \hline 5 & $7.2582 \mathrm{e}-03$ & $3.5061 \mathrm{e}-03$ & 1.15 & 1.14 \\
6 & $7.6215 \mathrm{e}-03$ & $1.5399 \mathrm{e}-03$ & 1.21 & 1.17 \\
7 & $8.0226 \mathrm{e}-03$ & $9.6963 \mathrm{e}-04$ & 1.27 & 1.12 \\
8 & $8.3386 \mathrm{e}-03$ & $6.7518 \mathrm{e}-04$ & 1.32 & 1.08 \\
9 & $8.6656 \mathrm{e}-03$ & $5.0212 \mathrm{e}-04$ & 1.37 & 1.03 \\
10 & $8.8650 \mathrm{e}-03$ & $3.7695 \mathrm{e}-04$ & 1.40 & 1.00 \\
11 & $9.0605 \mathrm{e}-03$ & $3.1170 \mathrm{e}-04$ & 1.43 & 0.95 \\
12 & $9.2223 \mathrm{e}-03$ & $2.5031 \mathrm{e}-04$ & 1.46 & 0.92 \\
13 & $9.4103 \mathrm{e}-03$ & $2.1119 \mathrm{e}-04$ & 1.49 & 0.89 \\
14 & $9.4945 \mathrm{e}-03$ & $1.8101 \mathrm{e}-04$ & 1.50 & 0.85 \\
15 & $9.6058 \mathrm{e}-03$ & $1.6113 \mathrm{e}-04$ & 1.52 & 0.82 \\
16 & $9.7010 \mathrm{e}-03$ & $1.3771 \mathrm{e}-04$ & 1.54 & 0.80 \\
17 & $9.7389 \mathrm{e}-03$ & $1.7754 \mathrm{e}-04$ & 1.54 & 0.71 \\
18 & $9.7754 \mathrm{e}-03$ & $1.0544 \mathrm{e}-04$ & 1.55 & 0.76 \\
19 & $9.8542 \mathrm{e}-03$ & $1.2798 \mathrm{e}-04$ & 1.56 & 0.69 \\
20 & $9.8902 \mathrm{e}-03$ & $5.5130 \mathrm{e}-02$ & 1.57 & 0.14 \\
\hline \hline
\end{tabular}

Table 5 Stability, $\zeta^{(s t a b)}$, and accuracy, $\zeta^{(a c c)}$, efficiencies of the optimized fourth-order explicit Runge-Kutta methods for the advection equation.

\begin{tabular}{|c|c|c|c|c|}
\hline \multirow[b]{2}{*}{$s$} & \multirow[b]{2}{*}{$\Delta t$} & \multirow[b]{2}{*}{$C^{(p+1)}$} & \multicolumn{2}{|c|}{ Comparison with ERKF $(6,5)$} \\
\hline & & & $\begin{array}{c}\zeta^{s t a b} \\
(\Delta t / s)_{E R K F(6,5)}=3.6732 \mathrm{e}-03\end{array}$ & $\zeta_{(C)_{E R K F(6,5)}^{(p+1)} \zeta^{a c c}}=3.3557 \mathrm{e}-03$ \\
\hline 7 & $4.1152 \mathrm{e}-03$ & $1.5340 \mathrm{e}-03$ & 1.12 & 1.00 \\
\hline 8 & $4.4558 \mathrm{e}-03$ & $9.4552 \mathrm{e}-04$ & 1.21 & 0.97 \\
\hline 9 & $4.7240 \mathrm{e}-03$ & $6.2796 \mathrm{e}-05$ & 1.29 & 1.48 \\
\hline 10 & $4.9236 \mathrm{e}-03$ & $1.2186 \mathrm{e}-04$ & 1.34 & 1.16 \\
\hline 11 & $5.0647 \mathrm{e}-03$ & $2.5424 \mathrm{e}-05$ & 1.38 & 1.45 \\
\hline 12 & $5.1825 \mathrm{e}-03$ & $1.9784 \mathrm{e}-05$ & 1.41 & 1.40 \\
\hline 13 & $5.2668 \mathrm{e}-03$ & $1.1512 \mathrm{e}-05$ & 1.43 & 1.44 \\
\hline 14 & $5.3464 \mathrm{e}-03$ & $3.0884 \mathrm{e}-05$ & 1.46 & 1.09 \\
\hline 15 & $5.4026 \mathrm{e}-03$ & $4.9569 \mathrm{e}-05$ & 1.47 & 0.93 \\
\hline 16 & $5.4610 \mathrm{e}-03$ & $4.8870 \mathrm{e}-05$ & 1.49 & 0.87 \\
\hline 17 & $5.5103 \mathrm{e}-03$ & $2.6513 \mathrm{e}-05$ & 1.50 & 0.93 \\
\hline 18 & $5.5592 \mathrm{e}-03$ & $8.9630 \mathrm{e}-06$ & 1.51 & 1.09 \\
\hline 19 & $5.6006 \mathrm{e}-03$ & $8.9630 \mathrm{e}-06$ & 1.52 & 1.03 \\
\hline 20 & $5.6400 \mathrm{e}-03$ & $1.0084 \mathrm{e}-05$ & 1.54 & 0.96 \\
\hline
\end{tabular}

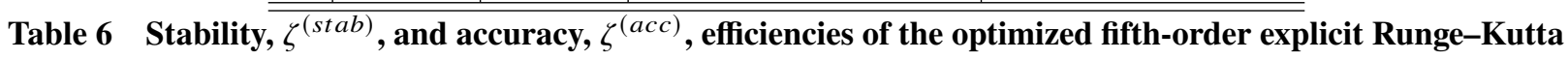
methods for the advection equation. 


\section{Acknowledgments}

The work described in this paper was supported by King Abdullah University of Science and Technology through the award OSR-2019-CCF-3666. The authors are also thankful for the computing resources of the Supercomputing Laboratory and the Extreme Computing Research Center at King Abdullah University of Science and Technology

\section{References}

[1] Hesthaven, J. S., Numerical methods for conservation laws: From analysis to algorithms, SIAM, 2017.

[2] Wang, Z., Fidkowski, K., Abgrall, R., Bassi, F., Caraeni, D., Cary, A., Deconinck, H., Hartmann, R., Hillewaert, K., Huynh, H., Kroll, N., May, G., Persson, P.-O., Leer, B., , and Visbal, M., "High-order CFD methods: current status and perspective," International Journal for Numerical Methods in Fluids, Vol. 72, No. 8, 2013, pp. 811-845.

[3] Abgrall, R., and Ricchiuto, M., "High-Order Methods for CFD," Encyclopedia of Computational Mechanics Second Edition, 2018, pp. 1-54.

[4] Cockburn, B., "Discontinuous Galerkin methods for computational fluid dynamics," Encyclopedia of Computational Mechanics Second Edition, 2018, pp. 1-63.

[5] Fischer, P., Min, M., Rathnayake, T., Dutta, S., Kolev, T., Dobrev, V., Camier, J.-S., Kronbichler, M., Warburton, T., and Swirydowicz, J., K. Brown, "Scalability of high-performance PDE solvers," The International Journal of High Performance Computing Applications, 2020, pp. 1-25.

[6] Hutchinson, M., Heinecke, A., Pabst, H., Henry, G., Parsani, M., and Keyes, D., "Efficiency of high order spectral element methods on petascale architectures," International Conference on High Performance Computing, 2016, pp. 449-466.

[7] Vincent, P., Witherden, F., Vermeire, B., Park, J. S., and Iyer, A., “Towards Green Aviation with Python at Petascale,” SC '16: Proceedings of the International Conference for High Performance Computing, Networking, Storage and Analysis, 2016, pp. $1-11$.

[8] Hadri, B., Parsani, M., Hutchinson, M., Heinecke, A., Dalcin, L., and Keyes, D., "Performance Study of Sustained Petascale Direct Numerical Simulation on Cray XC40 Systems (Trinity, Shaheen2 and Cori)," Concurrency and Computation: Practice and Experience, 2020.

[9] Merzari, E., Fischer, P., Min, M., Kerkemeier, S., Obabko, A., Shaver, D., Yuan, H., Yu, Y., Martinez, J., Brockmeyer, L., Fick, L., Busco, G., Yildiz, A., and Hassan, Y., "Toward Exascale: Overview of Large Eddy Simulations and Direct Numerical Simulations of Nuclear Reactor Flows with the Spectral Element Method in Nek5000," Nuclear Technology, 2020, pp. 1-17.

[10] Tadmor, E., "Entropy stability theory for difference approximations of nonlinear conservation laws and related time-dependent problems," Acta Numerica, Vol. 12, 2003, pp. 451-512.

[11] LeFloch, P. G., Mercier, J.-M., and Rohde, C., "Fully discrete, entropy conservative schemes of arbitrary order," SIAM Journal on Numerical Analysis, Vol. 40, No. 5, 2002, pp. 1968-1992.

[12] Fjordholm, U. S., Mishra, S., and Tadmor, E., "Arbitrarily high-order accurate entropy stable essentially nonoscillatory schemes for systems of conservation laws," Communications in Computational Physics, Vol. 50, No. 2, 2012, pp. 554-573.

[13] Carpenter, M. H., Fisher, T. C., Nielsen, E. J., and Frankel, S., "Entropy stable spectral collocation schemes for the Navier-Stokes equations: Discontinuous interfaces," SIAM Journal on Scientific Computing, Vol. 36, No. 5, 2014, pp. 835-867.

[14] Parsani, M., Carpenter, M. H., and Nielsen, E. J., "Entropy stable wall boundary conditions for the three-dimensional compressible Navier-Stokes equations," Journal of Computational Physics, Vol. 292, 2015, pp. 88-113. 
[15] Gassner, G. J., Winters, A. R., and Kopriva, D. A., "Split form nodal discontinuous Galerkin schemes with summation-by-parts property for the compressible Euler equations," Journal of Computational Physics, Vol. 327, 2016, pp. $39-66$.

[16] Chen, T., and Shu, C. W., "Entropy stable high order discontinuous Galerkin methods with suitable quadrature rules for hyperbolic conservation laws," Journal of Computational Physics, Vol. 345, 2017, pp. 427 - 461.

[17] Crean, J., Hicken, J. E., Del Rey Fernández, D. C., Zingg, D. Z., and Carpenter, M. H., “Entropy-stable summation-by-parts discretization of the Euler equations on general curved elements," Journal of Computational Physics, Vol. 356, 2018, pp. 410 -438 .

[18] Chan, J., "On discretely entropy conservative and entropy stable discontinuous Galerkin methods," Journal of Computational Physics, Vol. 362, 2018, pp. 346-374.

[19] Sjörn, B., and Yee, H. C., "High order entropy conservative central schemes for wide ranges of compressible gas dynamics and MHD flows," Journal of Computational Physics, Vol. 364, 2018, pp. 153-185.

[20] Abgrall, R., Nordström, J., Öffner, P., and Tokareva, S., "Analysis of the SBP-SAT Stabilization for Finite Element Methods Part II: Entropy Stability,” arXiv preprint arXiv:1912.08390, 2019.

[21] Hicken, J. E., "Entropy-Stable, High-Order Summation-by-Parts Discretizations Without Interface Penalties," Journal of Scientific Computing, Vol. 82, 2020.

[22] Rojas, D., Boukharfane, R., Dalcin, L., Fernández, D. C. D. R., Ranocha, H., Keyes, D. E., and Parsani, M., “On the robustness and performance of entropy stable collocated discontinuous Galerkin methods," Journal of Computational Physics, 2020 , p. 109891.

[23] Wu, K., and Shu, C.-W., "Entropy symmetrization and high-order accurate entropy stable numerical schemes for relativistic MHD equations," SIAM Journal on Scientific Computing, Vol. 42, No. 4, 2020, pp. A2230-A2261.

[24] Parsani, M., Boukharfane, R., Nolasco, I. R., Del Rey Fernández, D. C., Zampini, S., Hadri, B., and Dalcin, L., "High-order accurate entropy-stable discontinuous collocated Galerkin methods with the summation-by-parts property for compressible CFD frameworks: Scalable SSDC algorithms and flow solver," Journal of Computational Physics, Vol. 424, 2021 , p. 109844.

[25] Niegemann, J., Diehl, R., and Busch, K., "Efficient low-storage Runge-Kutta schemes with optimized stability regions," Journal of Computational Physics, Vol. 231, No. 2, 2012, pp. 364-372.

[26] Ketcheson, D. I., and Ahmadia, A., "Optimal stability polynomials for numerical integration of initial value problems," Communications on Applied Mathematics and Computation, Vol. 7, No. 2, 2012, pp. 247-271.

[27] Parsani, M., Ketcheson, D. I., and Deconinck, W., "Optimized Explicit Runge-Kutta Schemes for the Spectral Difference Method Applied to Wave Propagation Problems," SIAM Journal on Scientific Computing, Vol. 35, No. 2, 2013, pp. A957-A986.

[28] Toulorge, T., and Desmet, W., "Optimal Runge-Kutta schemes for discontinuous Galerkin space discretizations applied to wave propagation problems,” Journal of Computational Physics, Vol. 231, No. 4, 2012, pp. 2067-2091.

[29] Kubatko, E. J., Yeager, B. A., and Ketcheson, D. I., "Optimal strong-stability-preserving Runge-Kutta time discretizations for discontinuous Galerkin methods," Journal of Scientific Computing, Vol. 60, No. 2, 2014, pp. 313-344.

[30] Vermeire, B. C., Loppi, N. A., and Vincent, P. E., “Optimal Runge-Kutta schemes for pseudo time-stepping with high-order unstructured methods," Journal of Computational Physics, Vol. 383, 2019, pp. 55-71.

[31] Butcher, J. C., Numerical Methods for Ordinary Differential Equations, John Wiley \& Sons Ltd, 2008. 
[32] Wanner, G., and Hairer, E., Solving ordinary differential equations II, Springer Berlin Heidelberg, 1996.

[33] Parsani, M., Carpenter, M. H., and Nielsen, E. J., "Entropy stable discontinuous interfaces coupling for the three-dimensional compressible Navier-Stokes equations," Journal of Computational Physics, Vol. 290, 2015, pp. 132-138.

[34] Parsani, M., Carpenter, M. H., Fisher, T., and Nielsen, E., "Entropy stable staggered grid discontinuous spectral collocation methods of any order for the compressible Navier-Stokes equations," SIAM Journal on Scientific Computing, Vol. 38, No. 5, 2016, pp. 3129-3162.

[35] Dalcin, L., Rojas, D., Zampini, S., Del Rey Fernández, D. C., Carpenter, M. H., and Parsani, M., "Conservative and entropy stable solid wall boundary conditions for the compressible Navier-Stokes equations: Adiabatic wall and heat entropy transfer," Journal of Computational Physics, Vol. 397, 2019, p. 108775.

[36] Del Rey Fernández, D. C., Carpenter, M. H., Dalcin, L., Fredrich, L., Rojas, D., Winters, A. R., Gassner, G. J., Zampini, S., and Parsani, M., "Entropy stable non-conforming discretizations with the summation-by-parts property for curvilinear coordinates," NASA TM-2020-220574, 2019.

[37] Parsani, M., Boukharfane, R., Nolasco, I., Del Rey Fernández, D. C., Zampini, S., Hadri, B., and Dalcin, L., "High-order accurate entropy-stable discontinuous collocated Galerkin methods with the summation-by-parts property for compressible CFD frameworks: Scalable SSDC algorithms and flow solver," Journal of Computational Physics, Vol. In press, 2020.

[38] Parsani, M., Carpenter, M. H., and Nielsen, E. J., "Entropy stable wall boundary conditions for the three-dimensional compressible Navier-Stokes equations,” Journal of Computational Physics, Vol. 292, No. 1, 2015, pp. 88-113.

[39] Del Rey Fernández, D. C., Carpenter, M. H., Dalcin, L., Zampini, S., and Parsani, M., "Entropy stable $h / p$ non-conforming discretization with the summation-by-parts property for the compressible Euler and Navier-Stokes equations," SN Partial Differential Equations and Applications, Vol. 1, No. 2, 2020, pp. 1-54.

[40] Chen, T., and Shu, C.-W., "Review of entropy stable discontinuous Galerkin methods for systems of conservation laws on unstructured simplex meshes,", 2020.

[41] Svärd, M., and Nordström, J., "Review of summation-by-parts schemes for initial boundary-value problems," Journal of Computational Physics, Vol. 268, 2014, pp. 17-38.

[42] Del Rey Fernández, D. C., Hicken, J. E., and Zingg, D. W., "Review of summation-by-parts operators with simultaneous approximation terms for the numerical solution of partial differential equations," Computers \& Fluids, Vol. 95, 2014, pp. $171-196$.

[43] Reyna Nolasco, I. E., Dalcin, L., Del Rey Fernández, D. C., Zampini, S., and Parsani, M., “Optimized geometrical metrics satisfying free-stream preservation,” Computers \& Fluids, 2020, p. 104555.

[44] Ketcheson, D. I., Parsani, M., Grant, Z. J., Ahmadia, A. J., and Ranocha, H., "RK-Opt: A package for the design of numerical ODE solvers, version 1.0.0,", July 2020. Version 1.0.0.

[45] Hairer, E., and Wanner, G., Solving Ordinary Differential Equations II: Stiff and Differential-Algebraic Problems, Vol. 14, Springer-Berlin Heidelberg, 2010.

[46] Ketcheson, D. I., "Runge-Kutta methods with minimum storage implementations," Journal of Computational Physics, Vol. 229, No. 5, 2010, pp. 1763-1773.

[47] Ketcheson, D. I., Parsani, M., Grant, Z. J., Ahmadia, A. J., and Ranocha, H., "RK-Opt: A package for the design of numerical ODE solvers," , 2020. 
[48] Sanz-Serna, J. M., and Spijker, M. N., "Regions of stability, equivalence theorems and the Courant-Friedrichs-Lewy condition," Numerische Mathematik, Vol. 49, No. 2-3, 1986, pp. 319-329.

[49] Stanescu, D., and Habashi, W. G., "2N-storage low dissipation and dispersion Runge-Kutta schemes for computational acoustics," Journal of Computational Physics, Vol. 143, No. 2, 1998, pp. 674-681.

[50] Niegemann, J., Diehl, R., and Busch, K., "Efficient low-storage Runge-Kutta schemes with optimized stability regions,” Journal of Computational Physics, Vol. 231, No. 2, 2011, pp. 372-364.

[51] Kopriva, D. A., Implementing Spectral Methods for Partial Differential Equations: Algorithms for Scientists and Engineers, Springer Science \& Business Media, New York, 2009.

[52] Butcher, J. C., Numerical methods for ordinary differential equations, John Wiley \& Sons, Ltd, Chichester, UK, 2008.

[53] Cenaero, "HiOCFD5, 5th International Workshop on High-Order CFD Methods,", 2018. URL https: //how5 . cenaero . be. 